\title{
Iatrogenic bile duct injuries during laparoscopic cholecystectomy
}

\author{
Aloka Pathirana, MS, FRCS - Convenor
}

\author{
Professor in Surgery, The University of Sri Jayawardenapura Medical School, Colombo, Sri Lanka
}

Key Words: Cholecystectomy, Bile duct injuries, Conversion

\section{Introduction}

Laparoscopic cholecystectomy (LC) is one of the most commonly performed surgical procedures by general surgeons worldwide. It is considered the "gold standard" for treatment of symptomatic gall stones [1]. With the introduction of the laparoscopic approach, there has been an increase in the incidence of surgical treatment of gall stones. Reasons for this could be; the operation seemingly being "straight forward", enthusiasm of surgeons to learn "novel procedures" and the obvious benefits to the patient in the form of early return to normalcy.

Like all other surgical procedures, LC too requires training, particularly because it involves learning of a different type of skill, where tactile sensation is replaced by magnified visibility of structures from a different perspective. After undergoing training either at a formal "hands on" course or by an experienced trainer, it is necessary to perform the operation under supervision. Difficulties encountered could be many it is necessary for the surgeon to be able to recognize that he/she is in a difficult situation and take steps to overcome, if not convert to the open method where, traditionally, one is "comfortable". It is also safer, for patient and surgeon, to resort to lesser procedures like cholecystostomy or subtotal cholecystectomy.

When LC was introduced a few decades ago, there was criticism by many, citing case series where the

Correspondence: Aloka Pathirana, Professor in Surgery, The University of Sri Jayawardenapura Medical School, Colombo, Sri Lanka

·Email: aloka@slt.lk

The Sri Lanka Journal of Surgery 2011; 29(2):77-78 incidence of mortality and morbidity was significant. At the time, a randomized trial comparing laparoscopic cholecystectomy to 'mini' cholecystectomy demonstrated no statistically significant benefit from LC [2]. Although LC is now considered the optimum treatment for symptomatic gall stones, there is enough evidence to suggest that this procedure is still causing significant morbidity and even mortality.

We have noted a steady, if not rising, incidence (an average of one referral per month during the year 2011) of iatrogenic bile duct injuries (IBDI). It is important to remember that the patient population that is offered this treatment is an otherwise healthy and productive part of society most are young females. Therefore, it is thought that addressing an issue as important as IBDI in the College journal, would increase awareness of this issue and help surgeons minimize IBDI.

\section{Reasons for IBDI 3 D's}

It has been noted that dangerous anatomy, dangerous pathology and dangerous surgery (or surgeon) account for IBDI [3].

Dangerous anatomy: It is important for surgeons to have a thorough understanding of anatomy in every single operation that is performed. In an area with such a wide variation of its anatomy, such as in the biliary tract, the place of such knowledge becomes even more emphatic. It would be ideal to have pre-operative knowledge of the anatomy. The incidence of anatomical abnormalities in the extra-hepatic biliary tree is noted be between $30-40 \%$ [4]. To assess the anatomy before operation, one would require a magnetic resonance choledochogram (MRCP). This is not a practical solution in any situation, and not necessary. Intra-operative cholangiography is appropriate and is practiced either selectively or in all 
cases. Although some authors mention that surgeons should be aware of all possible anatomical variations, we feel it may not be completely true. This is amply demonstrated a published case series of IBDI, where most injuries occurred when the anatomy of the biliary tree was considered to be normal [5]. The principles to follow are staying close to the gall bladder during dissection and not dividing any structure until identified.

Dangerous pathology: This is important particularly when operating on patients with a history of acute or chronic inflammation of the GB. This could result in fore- shortening of the cystic duct and difficult dissection in Calot's triangle. Such a situation should be recognized early and converted to open surgery if needed. Dangerous pathology is probably the only instance where the surgeon may be excused for causing IBDI.

Dangerous surgery (or surgeon): Several factors may be contributory; commencing from access (whether open or Veress needle), quality of the instruments (electrical injury), and the skill of the surgeon - all are of equal importance. Supervised training, in a systematic manner, would be most important to minimize such complications.

\section{Late conversion - the scourge}

The conversion rate for LC ranges from $15-20 \%$ [5]. Skilled surgeons argue that it is possible to perform almost any open operation by the laparoscopic route. However, we believe that these are prophecies of "skilled surgeons"- a very select group. It is important to be aware of one's capabilities. When LC is not progressing well it is essential to let go of one's "ego" (which we all have to varying extents), remember patient safety and to convert. This makes life much easier for the surgeon for, even if IBDI has occurred, at least, one is perceived as "safe".

There have been several guidelines for conversion. Inability to dissect out the contents of Calot's triangle within 20 minutes is generally considered an important factor predicting a difficult LC. Also, there are several objective indicators for early conversion. These include:

1. Pre-operative-history of acute cholecystitis, Obesity, male sex, thick walled gall bladder, pain or rigidity in the upper abdomen [6].

2. Intra-operative - dense adhesions to the gall bladder or adhesions in Calot's triangle and acute gall bladder inflammation.

The articles we have compiled have been drawn from experts. They cover all aspects relevant to a surgeon embarking on safe LC, ranging from how to avoid injury, to early identification and management. We sincerely hope that this series would be helpful in contributing to reduction in IBDI in our country.

\section{References}

1. Soper NJ, Stockmann PT, Dunnegan DL et al. Laparoscopic Cholecystectomy The New 'Gold Standard'? Arch Surg. 1992;127(8):917-923.

2. Majeed AW, Troy G, Nicholl JP et al. Randomized prospective single-blind comparison of laparoscopic and small incision cholecystectomy. Lancet 1996; 347: 989-994

3. Johnston GW. Iatrogenic bile duct injuries: an avoidable surgical hazard? British Journal of Surgery 1986;73:246-7

4. Poston GJ, Blumgart LH. Surgical anatomy of the liver and bile ducts In: : Poston GJ, Blumgart LH ed. Surgical Management of hepatobiliary and pancreatic disorders. London: Martin Dunitz 2003; $1-18$

5. Majeed AW, Johnson AG. Pitfalls in cholecystectomy In: : Poston GJ, Blumgart LH ed. Surgical Management of hepatobiliary and pancreatic disorders. London: Martin Dunitz 2003; 301-314

6. Kama NA, Kologlu M, Doganay M et al. A risk score for conversion from laparoscopic to open cholecystectomy American Journal of Surgery: $181 ; 520-525$ 